PHYSICAL REVIEW A 95, 019903(E) (2017)

\title{
Erratum: Scalable orbital-angular-momentum sorting without destroying photon states [Phys. Rev. A 94, 033847 (2016)]
}

Fang-Xiang Wang, Wei Chen, Zhen-Qiang Yin, Shuang Wang, Guang-Can Guo, and Zheng-Fu Han (Received 6 January 2017; published 31 January 2017)

DOI: 10.1103/PhysRevA.95.019903

A universal method for sorting high-dimensional quantum states has previously been published [1], while the sorter proposed in our paper can been regarded as a specific and optimized implementation of this method for orbital-angular momentum (OAM).

This work should have been cited in our paper, and we would like to thank Radu Ionicioiu for bringing this important work to our attention.

[1] R. Ionicioiu, Sci. Rep. 6, 25356 (2016). 